\title{
Thermal and Mechanical Analysis of Butt Welding
}

\author{
Heny Andya, Ling-Chieh Shen, Yuan-Zhang Luo, Chih-Kuang Lin*, Chih-Han Wu, Yi-Mei Huang \\ Department of Mechanical Engineering, National Central University \\ 300 Jhong-Da Rd., Jhong-Li District, Tao-Yuan, Taiwan \\ *t330014@cc.ncu.edu.tw
}

\section{Extended Abstract}

The objective of this study is to develop a computer aided engineering (CAE) technique through finite element method (FEM) for calculating temperature and stress distributions in butt welding of large plates. From the simulation results, it is accessible to predict residual stress distribution so as to assess the structural integrity of large-plate weldments. Experimental measurements of temperature are also carried out to validate the FEM simulation. A double ellipsoidal volumetric heat source is employed in the FEM model to simulate large-plate welding. Simulations of temperature variation at selected positions are compared with experimental measurements to verify the effectiveness of the FEM modeling technique developed. The thermal history at selected positions shows a similar trend between simulations and experiments. In particular, simulations of temperature variation highly concur with one of the three given experiments. After the welding and cooling process, calculated von-Mises equivalent stress at the heat affected zone (HAZ) is much larger than that at the outer region. The maximum residual stress is located at the melt pool area and has a value close to the yield strength of material. Therefore, plastic deformation is expected to take place around this highly stressed region. The magnitude of residual stress decreases with increasing distance from the melt pool area and HAZ. Higher compressive residual stress is concentrated at the starting and ending regions on the welding path, for the normal stress component in the direction perpendicular to the welding path. For the normal stress component in the direction parallel to the welding path, larger tensile residual stress is located at the melt pool area and it becomes compressive stress at the region away from the welding path. Hopefully, the developed CAE technique and validated FEM model could help plan a better welding process for large-plate welding and improve its structural robustness. 